\title{
Phase I/II study of weekly paclitaxel and carboplatin with concurrent radiation therapy in locally advanced non- small-cell lung cancer: Kansai Clinical Oncology Group T0401
}

\author{
Kazufumi Takamatsu' ${ }^{1 *}$, Satoshi Marumo ${ }^{1}$, Takehisa Takagi ${ }^{2}$, Motonari Fukui ${ }^{1}$, Kiyoshi Komuta ${ }^{3}$, Tadashi Mio $^{4}$ and Masataka Hirabayashi $^{5}$ \\ ${ }^{1}$ Respiratory Disease Centre, Division of Respiratory Medicine, Tazuke Kofukai Medical Institute Kitano Hospital, 2-4-20 Ohgimachi, Kita-ku, Osaka 530-8480, \\ Japan \\ ${ }^{2}$ Department of radiotherapy, Tazuke Kofukai Medical Institute Kitano Hospital, 2-4-20 Ohgimachi, Kita-ku, Osaka 530-8480, Japan \\ ${ }^{3}$ Division of Respiratory Medicine, Osaka Police Hospital,10-31 Kitayama Tennoji-ku Osaka 543-0035, Japan \\ ${ }^{4}$ Division of Respiratory Medicine, National Hospital Organization Kyoto Medical Centre, 1-1 Fushimi-ku Kyoto 612-8555, Japan \\ ${ }^{5}$ Division of Respiratory Medicine, Hyogo Prefectural Amagasaki Hospital, 2-17-77 Higashinanba Amagasaki-shi Hyogo 660-8550, Japan
}

\begin{abstract}
Background: Concurrent chemoradiotherapy (CCRT) is effective in patients with unresectable stage III locally advanced non-small-cell lung cancer (NSCLC). Weekly carboplatin and paclitaxel is a common CCRT regimen, but with variable results. Phase I of this study evaluated the recommended paclitaxel dosage. Phase II assessed response rates, progression-free survival, overall survival and adverse events.

Materials and Methods: A high, monthly single dose of carboplatin instead of a weekly divided dose was administered, and the dose intensity was increased. In the phase I study, patients with stage III NSCLC were treated with two cycles of four weeks of $40-70 \mathrm{mg} / \mathrm{m}^{2}$ intravenous paclitaxel on days 1 , 8 and 15 with carboplatin (area under the concentration curve (AUC 5) on day 1, and concurrent radiation therapy of 60 Gy in 30 fractions (2 Gy per fraction, 5 fractions per week). The phase I study results determined the phase II paclitaxel dose. The phase II primary endpoint was the CCRT response rate and secondary endpoints were progression-free survival, overall survival and safety.

Results: Phase I enrolled 12 patients. Because of dose-limiting toxicities at $50 \mathrm{mg} / \mathrm{m}^{2}$, the recommended paclitaxel dose was $40 \mathrm{mg} / \mathrm{m}^{2}$. Phase II enrolled 19 patients with a median age of 66 (range 54-74) years, 17 were men, 11 had adenocarcinoma, 7 had squamous cell carcinoma and one had adenosquamous carcinoma. The response rate was $91.7 \%$, progression-free survival was 11.7 months, and overall survival was 22.5 months. The incidence of grade 3 and 4 neutropaenia was $33 \%$ that of grade 2 or higher radiation pneumonitis was $15 \%$.
\end{abstract}

Conclusion: Concurrent chemoradiation therapy with monthly carboplatin (AUC 5) and weekly paclitaxel (40 mg/m²) might be effective and feasible for locally advanced NSCLC.

\section{Introduction}

Lung cancer is the leading cause of cancer-related death worldwide [1], and non-small cell lung cancer (NSCLC) accounts for $85 \%$ of all lung cancer cases [2]. Approximately $30 \%$ of patients with NSCLC present with locally advanced cancer [3]. Surgery is recommended for treatment stage III locally advanced NSCLC, but there are few resection candidates. In most unresectable cases, the standard treatment is combined thoracic radiotherapy (TRT) and chemotherapy [4-7]. Some randomised phase III studies of the timing of chemotherapy and radiotherapy reported that concurrent chemoradiation therapy (CCRT) significantly extended survival compared with sequential chemoradiation $[8,9]$.

National Comprehensive Cancer Network (NCCN) Guidelines recommend platinum-doublet chemotherapy during CCRT. Weekly paclitaxel and carboplatin is a frequently used regimen, but does not always have favourable outcomes compared with other regimens [10]. Because carboplatin reportedly has a dose-intensity relation [11], we, the Kansai Clinical Oncology Group (KCOG), hypothesised that a regimen with weekly low-dosage paclitaxel and monthly full dosage carboplatin would have enhanced anti-tumour effects. We conducted a phase I study to determine the recommended dosage of paclitaxel, and a phase II study to assess the efficacy and toxicity of this CCRT.

Correspondence to: Kazufumi Takamatsu, Department of Respiratory medicine, Kitano Hospital, The Tazuke Kofukai Medical Research Institute, 2-4-20 Ohgimachi, Kita-ku, Osaka 530-8480, Japan, Tel +81 66312 8824; Fax: +81 6 6312 8867; Email: jm-ktakamatsu@kochi-u.ac.jp

Key words: concurrent chemoradiotherapy, third-generation chemotherapy, dosedependency, locally advanced lung cancer

Received: July 22, 2017; Accepted: August 11, 2017; Published: August 14, 2017 


\section{Material and methods}

\section{Eligibility}

Patients with histologically or cytologically confirmed and unresectable stage III NSCLC were eligible for inclusion. Unresectable stage IIIA disease was defined by the presence of multiple or bulky N2 mediastinal lymph nodes on computed tomography (CT) or positron emission tomography (FDG-PET). Eligible patients also had no prior history of chemotherapy or TRT, were Eastern Cooperative Oncology Group (ECOG) performance status 0 or $1, \geq 20$ and $<75$ years of age, had leucocytes $\geq 4,000 / \mathrm{mm}^{3}$, neutrophils $\geq 2,000 / \mathrm{mm}^{3}$, platelets $\geq 100,000 /$ $\mathrm{mm}^{3}$, haemoglobin $\geq 9.0 \mathrm{~g} / \mathrm{dL}$, serum creatinine $\leq 1.5 \mathrm{mg} / \mathrm{dl}$, AST and ALT $\leq$ twice the upper limit of normal, partial pressure of arterial oxygen $>70 \mathrm{mmHg}$ and no abnormalities on echocardiographic evaluation.

Patients with pulmonary fibrosis requiring oxygen therapy, myocardial infarction within the previous 6 months, liver cirrhosis, active haemorrhage of the digestive tract, a mental disorder requiring treatment, poorly controlled diabetes mellitus, paralytic ileus active infection, a history of radiation in the fields treated in this study, allergy to components of polyoxyethylene preparations, peripheral neuropathy, or pregnancy were excluded. Patients judged by a physician to be unable to participate were also excluded. The study protocol was approved by the ethics committee of the Tazuke Kofukai Medical Research Institute, Kitano Hospital and all other participating institutions. The study was conducted following the ethical guidelines of the Declaration of Helsinki. All patients gave written informed consent before enrolment. The period of registration and follow-up was from April 2005 to May 2011. This study was registered with the UMIN Clinical Trials Registry, receipt no. R000030549, ID. UMIN000026606.

\section{Study design}

Treatment included initial concurrent chemoradiotherapy and subsequent consolidation chemotherapy. The phase I study was conducted to determine the recommended paclitaxel dosage. Chemotherapy began with paclitaxel at the designated dosage on days $1,8,15,29,36$ and 43 , and carboplatin (AUC $5 \mathrm{mg} / \mathrm{mL} / \mathrm{min}$ ) on days 1 and 29. The initial paclitaxel dose was $40 \mathrm{mg} / \mathrm{m}^{2}$ i.e. dose escalation level 1(Figure 1). On day 1 of chemotherapy, TRT was begun at a dosage of $2 \mathrm{~Gy} /$ fraction given five times weekly for 6 weeks (total $60 \mathrm{~Gy}$ ). We evaluated the response to this therapy at $40 \mathrm{~Gy}$ and considered the indication of curative surgery. If three patients did not have dose-limiting toxicity (DLT), then the subsequent three patients were administered paclitaxel at the next dosage escalation level. If DLT occurred in one patient, then three patients were added. If DLT occurred in two patients at the same dose escalation level, then that dose was the maximum tolerated dose (MTD) and the level below the MTD was the recommended dosage. If MTD did not occur until dose escalation level four, then the recommended dosage was $70 \mathrm{mg} /$ $\mathrm{m}^{2}$. For consolidation chemotherapy, paclitaxel was fixed at $60 \mathrm{mg} / \mathrm{m}^{2}$ on days 1,8 and 15, and carboplatin (AUC $5 \mathrm{mg} / \mathrm{mL} / \mathrm{min}$ ) on day 1 . Consolidation chemotherapy was repeated every 4 weeks, for two or more courses (Figure 1). Treatment after consolidation chemotherapy was not defined.

In the phase II study, paclitaxel was administered at the recommended dosage determined in the phase I study during concurrent chemoradiotherapy. Consolidation chemotherapy was conducted in the same way as in the phase I study. If DLT occurred, then paclitaxel was decreased by $10 \mathrm{mg} / \mathrm{m} 2$ in the next course in both phase I and II studies. At the start of the next course, chemotherapy was postponed unless leucocytes $\geq 3,000 / \mathrm{mm} 3$, neutrophils $\geq 1,500 / \mathrm{mm} 3$, platelets $\geq 100,000 / \mathrm{mm} 3$, haemoglobin $\geq 9.0 \mathrm{~g} / \mathrm{dL}$, serum creatinine $\leq$ $1.5 \mathrm{mg} / \mathrm{dl}$, AST and ALT $\leq$ twice upper limit of normal, ECOG PS was 0 or 1 , and non-haematologic toxicity was $\leq$ grade 2 .

\section{Radiation therapy}

All patients were treated with a linear accelerator photon beam of 4 MVX or more. The primary tumour and involved nodal disease received $60 \mathrm{~Gy}$ in 2-Gy fractions over 6 weeks. Radiation doses were specified at the centre of the target volume. Planning target volume 1 (PTV1) was calculated considering the clinical target volume and error in the reproducibility of respiratory movement. Planning target volume 2 (PTV2) was calculated in anticipation of subclinical extension (a margin of about $0.5 \mathrm{~cm}$ ) in gross target volume. The initial $40 \mathrm{~Gy}$ was delivered to planning target volume 1 (PTV1). The final $20 \mathrm{~Gy}$ was delivered to a reduced volume defined as planning target volume 2 (PTV2). PTV1 included the primary tumour, ipsilateral hilum and mediastinal nodal areas from the superior border of the aortic arch to $2-3 \mathrm{~cm}$ below the bifurcation of the trachea. The contralateral hilum was not included in PTV1. The index of the permitted maximum dose to normal tissue was planned so that the volume of the normal lung that irradiated the spinal cord was $44 \mathrm{~Gy}$ or less, and less than $30 \%$ that the lung was irradiated with more than 20 Gy. TRT was interrupted in patients with grade 4 haematologic toxicity, grade 3 to 4 oesophagitis

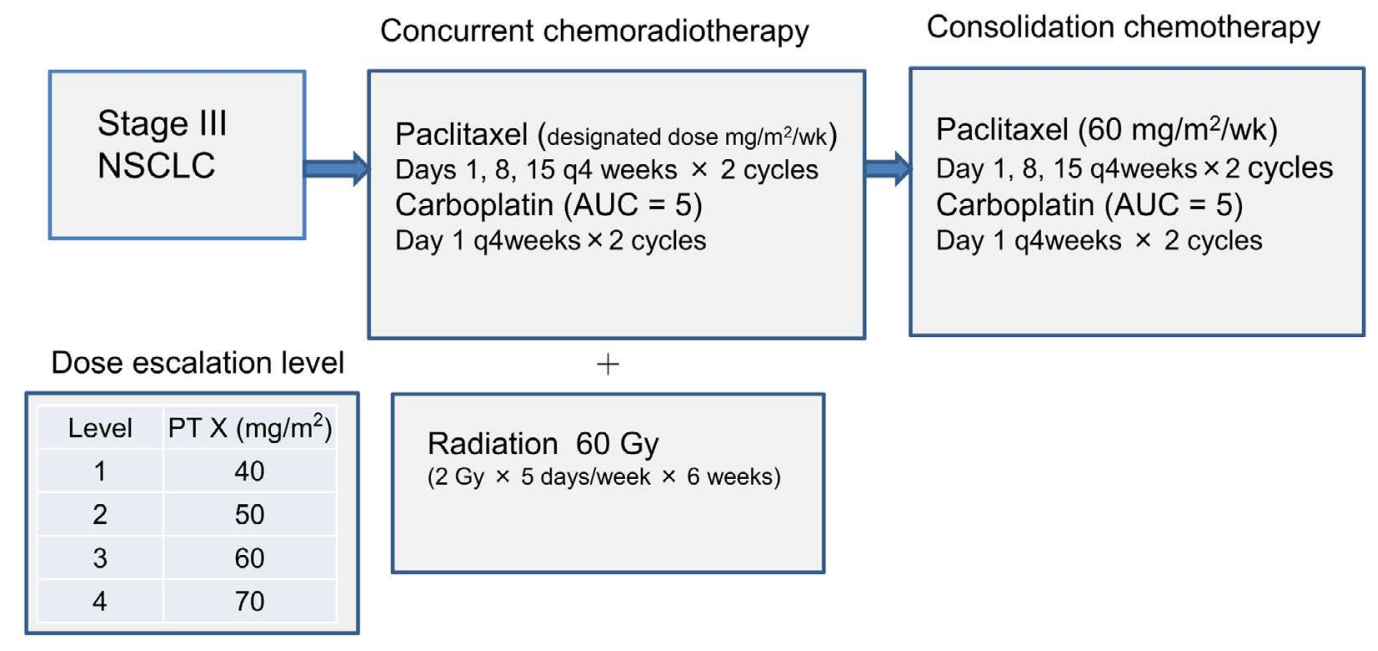

Figure 1. Design of study phase I. 
or dermatitis, pyrexia of $38^{\circ} \mathrm{C}$ or more, grade $2-4$ pneumonitis, or an arterial oxygen partial pressure of less than $60 \mathrm{mmHg}$.

\section{Efficacy and toxicity evaluation}

All eligible patients were evaluated for treatment response and toxicity. Complete blood counts (CBCs) and blood chemistry studies were repeated weekly during treatment. Chest X-rays, thoracic computed tomography (CT) and tumour markers were evaluated monthly during treatment. After treatment, imaging studies including fludeoxyglucose positron emission tomography (FDG-PET) and brain magnetic resonance imaging (MRI) were done if recurrence was suspected. Treatment response was evaluated by the Response Evaluation Criteria in Solid Tumours (RECIST) and response rates (RRs) were expressed as percentages of patients with complete response (CR) and partial response (PR). Disease control was reported as CR, PR and percentage of patients with stable disease (SD). Overall survival (OS) was the time from registration until death from any cause. Progression-free survival (PFS) was the time between registration and disease progression, death, or last known follow-up.

\section{Statistical methods}

The primary end point of the phase I study was the recommended dosage of paclitaxel during concurrent chemoradiotherapy. The primary endpoint of the phase II study was the treatment response rate; secondary endpoints were PFS, OS and safety. Evaluation of the feasibility of curative surgery was conducted following a cumulative 40 Gy radiation dose. Surgical cases were excluded from the evaluation of treatment effectiveness and toxicity. Patients who received the recommended phase I study dosage were included in the assessment of effectiveness. The response to concurrent chemoradiotherapy for unresectable locally advanced lung cancer has been estimated at $71 \%$ $78.6 \%$ [12-14]. When the lower limit of the expected response rate was $71 \%, 36$ cases were required. Considering dropouts, the planned study registration was 40 patients in the phase II study. Cumulative OS and PFS were estimated by the Kaplan-Meier method. Statistical analysis was conducted with StatMate IV (ATMS Co. Ltd., Tokyo, Japan).

\section{Results}

\section{Phase I study}

A total of 12 patients, nine men and three women were included in the phase I study. The patient characteristics are shown in Table 1 . The median age was 64 (43-74) years, three patients were stage IIIA, nine were stage IIIB, six patients were diagnosed with adenocarcinoma and the others with squamous cell carcinoma. DLT occurred at paclitaxel dose of $50 \mathrm{mg} / \mathrm{m}^{2}$, which was designated as the MTD. DLT occurred in two patients. One had grade 4 neutropaenia, the other had to skip chemotherapy two or more times to receive the planned administration dosage. Therefore, the recommended concurrent chemotherapy paclitaxel dosage was $40 \mathrm{mg} / \mathrm{m}^{2}$.

\section{Phase II study}

Recruitment was stopped at 5 years from the start of the study even though the planned enrolment had not been reached. A total of 19 patients were registered in the phase II study. The patient characteristics are shown in Table 1 . Seventeen men and two women were included, the median age was 66 (54-74) years, 9 patients were stage IIIA; 10 were stage IIIB, 11 were diagnosed with adenocarcinoma, seven with squamous cell carcinoma and one with adenosquamous cell carcinoma. Three patients were excluded because they were ultimately treated with surgery. The RR of the phase II study was $93.8 \%$ [95\% confidence interval (CI), $82-100 \%$ ]. The disease control rate was $100 \%$. If the eight patients administered the recommended dosage of paclitaxel in the phase I study were included in this analysis, then the response rate would be $91.8 \%$ (95\% CI, 80.9-100\%) and the disease control rate would be $100 \%$ (Table 2). The secondary endpoints, OS and median PFS, are shown in Fig. 2. Median survival time (MST) was 24.0 months; PFS was 14.8 months. When the eight patients in the phase I study were included, MST was 22.5 months and PFS 11.7 months. The three-year survival rate was $33.0 \%$.

Grade 3 or more severe toxicities included neutropaenia, leucopenia, thrombocytopenia, radiation pneumonitis, infection and peripheral neuropathy (Table 3). A treatment-related death of a 70-year-old man who was hospitalised with methicillin-resistant Staphylococcus aureus (MRSA) pneumonia on day 1 in the third course of chemotherapy was reported. The patient did not respond to antibiotics and eventually died. Nineteen of 27 patients (70\%) completed the study. Delay of starting treatment or skipping treatment administration occurred in 12 patients.

\section{Discussion}

The study demonstrated a good treatment response to and satisfactory tolerability of quad-weekly carboplatin (AUC 5) and weekly paclitaxel $\left(40 \mathrm{mg} / \mathrm{m}^{2}\right)$ combined with concurrent TRT of $60 \mathrm{~Gy}$ followed by quad-weekly carboplatin (AUC 5) and weekly paclitaxel (60 $\left.\mathrm{mg} / \mathrm{m}^{2}\right)$ in patients with NSCLC. In the phase I study, the appropriate dosage of paclitaxel was $40 \mathrm{mg} / \mathrm{m}^{2}$. In the phase II study, RR was $93.8 \%$; PFS was 11.7 months, and OS was 24 months. The most common grade 3 or more severe adverse event was neutropaenia, which was observed in $33.0 \%$ of patients.

Paclitaxel and carboplatin-including CCRT regimens for NSCLC used in previous studies are shown in Table 4. Decreasing doses of

\begin{tabular}{|c|c|c|c|c|}
\hline \multirow{2}{*}{ Characteristic } & \multicolumn{2}{|c|}{ Phase I $(\mathrm{N}=12)$} & \multicolumn{2}{|c|}{ Phase II (N = 19) } \\
\hline & No. & & No. & \\
\hline \multicolumn{5}{|l|}{ Sex } \\
\hline Female & 3 & 25 & 2 & 11 \\
\hline Male & 9 & 75 & 17 & 89 \\
\hline \multicolumn{5}{|l|}{ Age, years } \\
\hline Female & \multicolumn{2}{|c|}{64} & \multicolumn{2}{|c|}{66} \\
\hline Male & \multicolumn{2}{|c|}{$43-74$} & \multicolumn{2}{|c|}{$54-74$} \\
\hline \multicolumn{5}{|l|}{ Stage } \\
\hline IIIA & 3 & 25 & 9 & 47 \\
\hline IIIB & 9 & 75 & 10 & 53 \\
\hline \multicolumn{5}{|l|}{ Histology } \\
\hline Adenocarcinoma & & & 11 & 58 \\
\hline Squamous cell ca. & $\begin{array}{l}6 \\
6\end{array}$ & $\begin{array}{l}50 \\
50\end{array}$ & 7 & 37 \\
\hline Other & 6 & 50 & 1 & 5 \\
\hline
\end{tabular}

Table 2. Response rate (RR) and disease control rate (DCR)

\begin{tabular}{|c|c|c|}
\hline & RR (95\%CI, \%) & DCR (\%) \\
\hline Phase II & $93.8(82.0-100)$ & 100 \\
\hline Phase I + Phase II & $91.8(80.9-100)$ & 100 \\
\hline & Grade $3(\%)$ & Grade 4 or higher (\%) \\
\hline Neutropaenia & $8(30)$ & $1(4)$ \\
\hline Leucopaenia & $5(19)$ & $1(4)$ \\
\hline Thrombocytopaenia & $1(4)$ & 0 \\
\hline Infection & $3(11)$ & $1(4)$ \\
\hline Peripheral neuropathy & $2(7)$ & 0 \\
\hline Oesophagitis & 0 & 0 \\
\hline Radiation pneumonitis & $4(15)$ & 0 \\
\hline
\end{tabular}


A

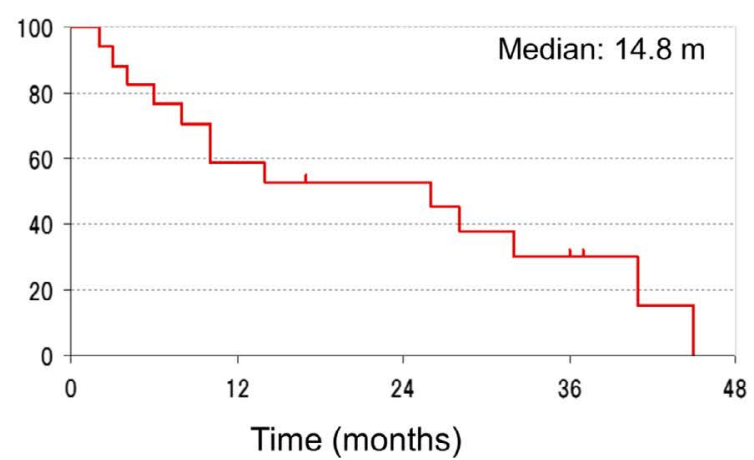

B

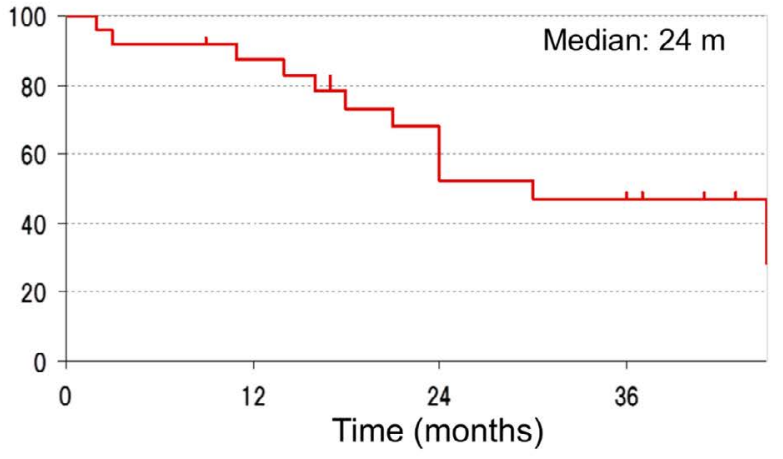

Figure 2. Progression-free and overall survival in study phase II. MST was 24.0 months; PFS was 14.8 months.

Table 4. Previous studies that used paclitaxel CCRT regimens

\begin{tabular}{|c|c|c|c|c|}
\hline & Treatment schedule & RR (\%) & MST (months) & 3-yr survival (\%) \\
\hline $\begin{array}{l}\text { RTOG } 9801 \\
\text { Phase III }\end{array}$ & $\begin{array}{l}\text { (1) } \mathrm{PC} \rightarrow \mathrm{PwCw}+\mathrm{Hf} \times \mathrm{RT}+\mathrm{AMO} \\
\text { (2) } \mathrm{PC} \rightarrow \mathrm{PwCw}+\mathrm{HfxRT}\end{array}$ & N.A & (1) 17.3 & $\begin{array}{l}\text { (1) } 28 \\
\text { (2) } 28\end{array}$ \\
\hline $\begin{array}{l}\text { WJTOG0105 } \\
\text { Phase III }\end{array}$ & $\begin{array}{l}\text { (1) } \mathrm{MVP}+\mathrm{XRT} \\
\text { (2) } \mathrm{CPT}-11+\mathrm{CBPw}+\mathrm{XRT} \\
\text { (3) } \mathrm{PwCw}+\mathrm{XRT}\end{array}$ & 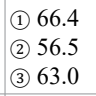 & 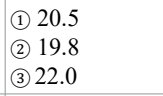 & $\begin{array}{l}\text { (1) } 35 \\
\text { (2) } 24 \\
\text { (3) } 26\end{array}$ \\
\hline $\begin{array}{l}\text { Luhua et al. } \\
\text { Randomized PhaseII }\end{array}$ & $\begin{array}{l}\text { (1) } \mathrm{PE} \\
\text { (2) } \mathrm{PwCw}\end{array}$ & (1) 63.7 & (1) 20.2 & $\begin{array}{l}\text { (1) } 33.1 \\
\text { (2) } 13\end{array}$ \\
\hline $\begin{array}{l}\text { KCOG T-0401 } \\
\text { Phase I/II }\end{array}$ & $\mathrm{PwC}+\mathrm{XRT}$ & 91.7 & 22.5 & 33 \\
\hline
\end{tabular}

carboplatin were administered weekly to augment radiosensitisation and to reduce adverse effects. WJTOG0105 was a phase III trial of concurrent chemoradiotherapy conducted in Japan to compare thirdand second-generation chemotherapy. No differences in median and 5-year-survival were seen in second- and third-generation chemotherapy arms, but a weekly paclitaxel and carboplatin regimen was proposed as standard CCRT because of its favourable toxicity profile in all three study arms. Previous reports show an RR of $63 \%$ to that regimen. The protocol used in this study resulted in a much better RR and better 3-year survival than reported in previous studies. These results might be explained by differences in the dosage and administration schedule of carboplatin followed in this study, which involved a higher quad-weekly single dose (AUC $=5$ on day 1$)$. In previous studies, the carboplatin dose was divided weekly (AUC $=2$ on days 1, 8 and 15). Duncan et al. have reported that the therapeutic response to carboplatin increased linearly up to doses of AUC 5-7 [11]. The CCRT response might thus be associated with the single carboplatin dose administered at each chemotherapy session. In this and most previous studies, divided low-dose administration of paclitaxel was used to enhance radiosensitisation and reduce the toxicities associated with CCRT [15-17]. Because DLT occurred with $50 \mathrm{mg} / \mathrm{m}^{2}$ paclitaxel in phase I of this study, the recommended dosage was set at $40 \mathrm{mg} / \mathrm{m}^{2}$, which was the same as used in WJTOG0105 and other studies.

In this study, most adverse effects were acceptable despite the single high carboplatin dose administered on days 1 and 29. Grade 3-4 haematological toxicity occurred in $37 \%$ of patients, which was a similar or lower frequency than previously reported [18-20]. Other studies such as WJTOG0105 found similar haematological toxicity in the concurrent phase, but increased haematological toxicity during consolidation therapy. Haematological toxicity did not increase during the consolidation phase of this study, which indicates that the regimen has acceptable safety compared with previous reports. Although recent reports have described $8.2 \%-36 \%$ of grade $3-4$ esophagitis [18-20], no incidence was found of grade 3-4 radiation esophagitisreported in this study. In previous studies, the incidence of grade 2 or higher radiation pneumonitis was $4.1 \%-10 \%$ and was often fatal. In this study, the incidence of grade 2 or worse radiation pneumonitis was $15 \%$, with no associated fatalities. Advances in radiotherapy such as fourdimensional CT and respiratory-gated radiotherapy can be expected to decrease the occurrence of radiation oesophagitis or radiation pneumonitis in future studies. Because $70 \%$ of the patients in this study completed consolidation chemotherapy, the tolerability of this regimen appears acceptable.

This study was limited by the small sample size. The planned enrolment in the phase II study was 40 patients, but reached less than half that number. The evaluation included the phase I study patients to increase the sample size. Consequently, eight patients given paclitaxel $40 \mathrm{mg} / \mathrm{m}^{2}$ were included. However, the RR $(93.8 \%$ in the phase II study and $91.8 \%$ in the phase I/II study), was not affected. The same result was seen for MST, which was 24.0 months in the phase I study and 22.5 months in the phase I/II study. Because there are many phase II studies of similar size, the subject size of the phase II study apparently poses no problem $[21,22]$.

In conclusion, CCRT with monthly carboplatin (AUC 5) and weekly paclitaxel $\left(40 \mathrm{mg} / \mathrm{m}^{2}\right)$ was an effective and tolerable regimen for these NSCLC patients. Increased dose-intensity may explain the effectiveness benefit of not dividing the carboplatin dose, but there were too few subjects to make a confident conclusion. A phase III study to compare this protocol with weekly carboplatin and weekly paclitaxel and TRT is warranted.

\section{Competing interests}

All authors have no competing interests. 


\section{Authors' contributions}

Kazufumi Takamatsu performed the statistical analysis and wrote the manuscript. Takehisa Takagi performed the radiotherapy and supervised the research of radiation part. Kiyoshi Komuta, Tadashi Mio and Masataka Hirabayashi collected the patients data. Satoshi Marumo and Motonari Fukui supervised the research and revised the manuscript. All authors have read and approved the final version of the manuscript.

\section{Acknowledgements}

This study was supported by Kansai Clinical Oncology Group. We are grateful to Hidetoshi Tokuhisa, M.D. who died in 2006 for planning and management as principal investigator.

\section{References}

1. Jemal A, Siegel R, Ward E, Murray T, Xu J, et al. (2007) Cancer statistics, 2007. CA Cancer J Clin 57: 43-66. [Crossref]

2. Toyoda Y, Nakayama T, Ioka A, Tsukuma H (2008) Trends in lung cancer incidence by histological type in Osaka, Japan. Jpn J Clin Oncol 38: 534-539. [Crossref]

3. van Meerbeeck JP (2001) Staging of non-small cell lung cancer: consensus, controversies and challenges. Lung Cancer 34 Suppl 2: S95-107. [Crossref]

4. Sause W, Kolesar P, Taylor S IV, Johnson D, Livingston R, et al. (2000) Final results of a phase III trial in regionally advanced unresectable non-small cell lung cancer: Radiation Therapy Oncology Group, Eastern Cooperative Oncology Group, and Southwest Oncology Group. Chest 117: 358-364. [Crossref]

5. Pritchard RS, Anthony SP (1996) Chemotherapy plus radiotherapy compared with radiotherapy alone in the treatment of locally advanced, unresectable, non-small-cell lung cancer. A meta-analysis. Ann Intern Med 125: 723-729. [Crossref]

6. Marino P, Preatoni A, Cantoni A (1995) Randomized trials of radiotherapy alone versus combined chemotherapy and radiotherapy in stages IIIa and IIIb nonsmall cell lung cancer. A meta-analysis. Cancer 76: 593-601. [Crossref]

7. Non-small Cell Lung Cancer Collaborative Group (1995) Chemotherapy in non-small cell lung cancer: A meta-analysis using update data on individual patients from 52 randomized clinical trials. BMJ 311: 899-909. [Crossref]

8. Furuse K, Fukuoka M, Kawahara M, Nishikawa H, Takeda Y, et al. (1999) Phase III study of concurrent vs. sequential thoracic radiotherapy in combination with mitomycin, vindesine, and cisplatin in unresectable stage III non-small cell lung cancer. J Clin Oncol 17:2692-2699. [Crossref]
9. Curran WJ, Scott C, Langer CJ, Komaki R, Lee JS, et al. (2011) Sequential vs. concurrent chemoradiation for stage III non-small cell lung cancer: randomized phase III trial RTOG 9410. J Natl Cancer Inst 103:1452-1460. [Crossref]

10. Yamamoto N, Nakagawa K, Nishimura Y, Tsujino K, Satouchi M, et al. (2010) Phase III study comparing second- and third-generation regimens with concurrent thoracic radiotherapy in patients with unresectable stage III non-small-cell-lung cancer. $J$ Clin Oncol 28:3739-3745. [Crossref]

11. Jodrell DI, Egorin MJ, Canetta RM, Langenberg P, Goldbloom EP, et al. (1992) Relationships between carboplatin exposure and tumor response and toxicity in patients with ovarian cancer. J Clin Oncol 10:520-528. [Crossref]

12. Lau D, Leigh B, Gandara D, Edelman M, Morgan R, et al. (2001) Twice-weekly paclitaxel and weekly carboplatin with concurrent thoracic radiation followed by carboplatin/paclitaxel consolidation for stage 3 non-small-cell lung cancer: A california cancer consortium phase II trial. J Clin Oncol 19:442-447. [Crossref]

13. Choy H, Akerley W, Safran H, Graziano S, Chung C, et al. (1998) Multiinstitutional Phase 2 trial of paclitaxel, carboplatin, and concurrent radiation therapy for locally advanced non-small-cell lung cancer. J Clin Oncol 16:3316-3322. [Crossref]

14. Isokangas OP, Joensuu H, Halme M, Jekunen A, Mattson K (1998) Paclitaxel (taxol) and carboplatin followed by concomitant paclitaxel, cisplatin and radiotherapy for inoperable stage 3 NSCLC. Lung Cancer 20:127-133. [Crossref]

15. Tishler RB, Geard CR, Hall EJ, Schiff PB (1992) Taxol sensitizes human astrocytoma cells to radiation. Cancer Res 52: 3495-3497. [Crossref]

16. Steren A, Sevin BU, Perras J, Angioli R, Nguyen H, et al. (1993) Taxol sensitizes human ovarian cancer cells to radiation. Gynecol Oncol 48: 252-258. [Crossref]

17. Choy H, Rodriguez FF, Koester S, Hilsenbeck S, Von Hoff DD (1993) Investigation of taxol as a potential radiation sensitizer. Cancer 71: 3774-3778. [Crossref]

18. Wang L, Wu S, Ou G, Bi N, Li W, et al. (2012) Randomized phase II study of concurrent cisplatin/etoposide or paclitaxel/carboplatin and thoracic radiotherapy in patients with stage III non-small cell lung cancer. Lung Cancer 77: 89-96. [Crossref]

19. Vokes EE, Herndon JE 2nd, Kelley MJ, Cicchetti MG, Ramnath N, et al (2007) Induction chemotherapy followed by chemoradiotherapy compared with chemoradiotherapy alone for regionally advanced unresectable stage III non-small-cell lung cancer. J Clin Oncol 25:1698-1704. [Crossref]

20. Movsas B, Scott C, Langer C, Werner Wasik M, Nicolaou N, et al. (2005) Randomized trial of amifostine in locally advanced non-small-cell lung cancer patients receiving chemotherapy and hyperfractionated radiation, Radiation Therapy Oncology Group Trial 98-01. J Clin Oncol 23: 2145-2154. [Crossref]

21. Ishida K, Hirose T, Yokouchi J, Oki Y, Kusumoto S, et al (2014) Phase II study of concurrent chemoradiotherapy with carboplatin and vinorelbine for locally advanced non-small-cell lung cancer. Mol Clin Oncol 2: 405-410. [Crossref]

22. Oshiro Y, Okumura T, Kurishima K, Homma S, Mizumoto M, et al. (2014) High-dose concurrent chemo-proton therapy for Stage III NSCLC: preliminary results of a Phase II study. J Radiat Res 55: 959-965. [Crossref]

Copyright: (C2017 Takamatsu K. This is an open-access article distributed under the terms of the Creative Commons Attribution License, which permits unrestricted use, distribution, and reproduction in any medium, provided the original author and source are credited. 